\title{
Structure Analysis and Decoupling Research of a Novel Flexible Tactile Sensor Array
}

\author{
Feilu Wang, Yang Song, Zhenya Zhang, and Wanli Chen \\ School of Electronics and Information Engineering, Anhui Jianzhu University, Hefei 230601, China \\ Correspondence should be addressed to Yang Song; esunny@ahjzu.edu.cn
}

Received 5 February 2015; Accepted 1 May 2015

Academic Editor: Sang Sub Kim

Copyright ( 2015 Feilu Wang et al. This is an open access article distributed under the Creative Commons Attribution License, which permits unrestricted use, distribution, and reproduction in any medium, provided the original work is properly cited.

\begin{abstract}
This paper presents a novel flexible tactile sensor structure and proposes an efficient decoupling algorithm for the tactile sensor. Firstly, structure of the sensor model is introduced, and the sensing mechanism of the sensor array based on force-sensitive conductive rubber is analyzed. Then the mapping relation between the resistances of conductive pillars and the three-dimensional force is deduced. After that, the force applied on the tactile sensor is decoupled from the resistance information by the improved Back Propagation Neural Network (BPNN) algorithm with the number of hidden layer nodes optimized. The flexible tactile sensor model achieves the decomposition of the three-dimensional information from the structure with its unique design, avoids the direct interference between electrodes of the sensor array, reduces the structural complexity and the nonlinear degree, improves the decoupling accuracy, and accelerates the decoupling rate.
\end{abstract}

\section{Introduction}

With the rapid development of intelligent robot technology, the research of tactile sensors has become a key issue in intelligent robot skin field. Tactile sensors can help robot to feel and recognize objects and to complete a variety of complex tasks. Particularly, the flexible tactile sensor is a very important part for robots to improve its intelligent level. It is required that the tactile senor should have softness and flexibility as human skin and be suitable for objects with different roughness and shape and can quickly and accurately achieve multi-dimensional information from surrounding environment. In practical, flexible tactile sensors are expected to be used as robot skin by which researchers want to detect the force loaded on the surface of objects. As sensitive tactile perception is critical for ensuring the safe and efficient interaction between robot and outside environment, the research of flexible tactile sensor plays an irreplaceable role in the bionic intelligent robot field.

Most of the design principle for the tactile sensor is mainly concentrated on MEMS [1], piezoelectricity [2], capacitor [3], PVDF [4], PDMS [5], optical fibers [6], elastic conductors technology [7], and so forth, from which researchers achieved a series of valuable accomplishments. Shimojo et al. [8] proposed a tactile sensor sheet using pressure conductive rubber with electrical-wires stitched method. That sensor is flexible and thin and can cover three-dimensional objects, and it is durable with respect to external force. Lipomi et al. [9] proposed skin-like pressure and strain sensors based on transparent elastic films of carbon nanotubes. The sensor can measure the deformation caused by a butterfly resting on the surface. Asadnia et al. [10] developed a highly stretchable, self-powered, and ultrasensitive strain sensor based on piezoelectricity PVDF nanofibers. That flexible strain sensor has good performance in human's motion recognition by fabricating a glove with two sensors mounted on the middle and index fingers. Engel et al. [11] developed a polyimide-based two-dimensional tactile sensing array using a novel inverted fabrication technique, which is low-cost, robust, and flexible micro-machined. Harada et al. [12] demonstrated a fully printed fingerprintlike three-axis tactile force and temperature sensor for artificial skin applications. The high sensitivity and selectivity for both force and temperature are demonstrated using a 3 $\times 3$ array artificial skin that senses tactile, slip/friction, and temperature. 


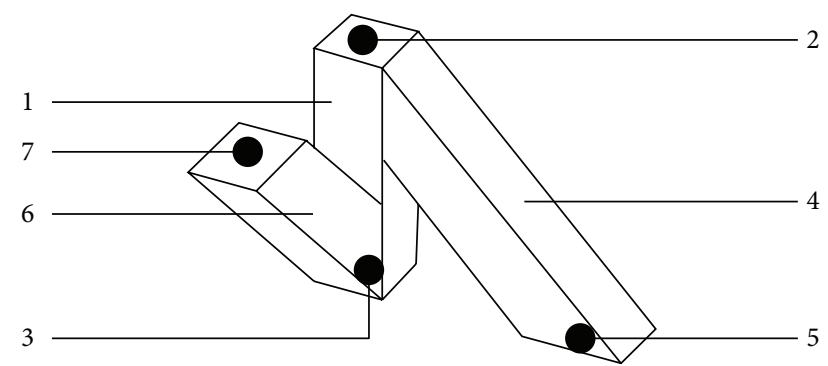

FIgURE 1: Micro structure sensitive unit.

As the coupling relation usually exists between the variables of tactile sensor array, decoupling algorithms for the sensor's high dimensional information are especially important in the design of tactile sensors. Wu et al. [13] proposed a static decoupling algorithm of six-axis force sensor based on coupling error and piecewise fitting model. Lee et al. [14] proposed a new type of slim, flexible tactile sensor. A triaxial force decoupling algorithm was developed by combining two-dimensional mapping data calculated by finite element analysis, which is able to measure the triaxial forces only using four strain gauges. Petra et al. [15] improved the distributed tactile sensor system based on BPNN and realized the decoupling circuit based on hardware equipment. Chen et al. [16] put forward a kind of data decoupling algorithm for the overvoltage sensor based on interphase coupling theory, which is simple and practical and has high precision. Baglio et al. [17] extracted the characteristic signal in the unknown surface of tactile sensor system based on the fuzzy logic. Ding et al. [18] presented a decoupling algorithm based on homotopy theory for three-dimensional tactile sensor array. In [19], the three-dimensional information of a tactile sensor is solved by artificial neural network methods under the ideal conditions. This paper takes full advantage of improved BPNN with its strong nonlinear approximation ability to decouple the high dimensional nonlinear mapping relation between resistance and force for the novel flexible tactile sensor.

\section{A Novel Flexible Tactile Sensor Model}

In order to improve the decoupling accuracy and real-time capability of the flexible tactile sensor, this paper presents a novel flexible tactile sensor model based on force-sensitive conductive rubber material. With its special structure, the three-dimensional force applied on the sensor can be decomposed directly, the complexity and nonlinear degree of the sensor are reduced, and the high dimensional information of the senor could be decoupled quickly and accurately.

2.1. Micro Structure Sensitive Unit. The core component of the novel flexible tactile sensor is the orderly arranged micro structure sensitive unit whose structure is shown in Figure 1. The three-dimensional micro structure is composed of three pillars made by force-sensitive conductive rubber. In view of the property of conductive rubber, the micro structure is flexible and very sensitive to three-dimensional force. In
Figure 1, "1," " 4 ," and " 6 " denote the three conductive pillars, respectively, and they are closely connected. "2" and " 7 " on the pillar labeled "1" and " 6 " denote the sensing electrode on the upper layer of the unit and " 3 " and " 5 " on the pillar labeled " 1 " and " 4 " denote the sensing electrode on the lower layer of the unit. The planes containing axes of pillars "4" and "6," respectively, are perpendicular to each other. Spaces between different micro structure sensitive units are filled up by flexible insulation material such as silicon rubber.

Initially, length of pillar 1 is $1 \mathrm{~cm}$ and lengths of pillar 4 and pillar 6 are both $\sqrt{2} \mathrm{~cm}$. Pillar 1 is perpendicular to the horizontal plane. The angle between pillar 1 and pillar 4 and that between pillar 1 and pillar 6 are both $45^{\circ}$. The crosssectional area of each pillar is $4 \mathrm{~mm}^{2}$.

2.2. Flexible Tactile Sensor Array. The novel tactile sensor array structure shown in Figure 2 is mainly composed of three parts. The first part is the sensing array that consists of three-dimensional sensitive units as shown in Figure 1. The second part is electrical wires that connect the micro structure sensitive units and the external acquisition circuit. The remaining part is the framework made by flexible insulation material (such as insulating rubber). The sensitive unit made by conductive rubber is the force-sensitive component of the novel flexible tactile sensor. In Figure 2, " 8 " denotes the electrical wires that connect the upper electrodes (such as "2" and "7"), it is called "row-wire"; "9" denotes the electrical wires that connect the lower electrodes (such as " 3 " and " 5 "); it is called "column-wire." The upper row-wires and the lower column-wires in two planes are perpendicular to each other.

When force exerted on the sensor, the tactile sensor transmits the three-dimensional force into the three conductive pillars separately. Resistance of the conductive pillar near the stress area will be changed, which can be detected by the external circuit of the sensor; then the three-dimensional force can be solved by the changed resistance of conductive pillars based on decoupling algorithms.

2.3. Detection Principle. Three-dimensional force loaded on the tactile sensor can be decomposed into three components based on its subtle structure design, which are expressed by $F_{x}, F_{y}$, and $F_{z}$, respectively. Almost each dimension of force component loaded on the sensor may affect the output signal. The insulating rubber near the stress area would be extruded by the loaded external force, and the force would be transmitted to the adjacent sensitive units, and then the length of conductive pillars could be changed. Meanwhile, the resistance of those pillars could be changed correspondingly. The resistances of pillars can be detected by the circuit through sequentially scanning the resistance between each row-wire in the upper layer and all of the column-wire in the lower layer. $R_{z}, R_{x}$, and $R_{y}$ denote the original resistance of the pillar labeled " 1 ," " 4 ," and "6".

As load usually loaded on the upper surface of the sensor has little influence on the lower surface and the lower surface of tactile sensor is usually attached to or fixed on the surface of robot and other objects, it is assumed that when force is applied on the sensor, the lower layer has no deformation, 


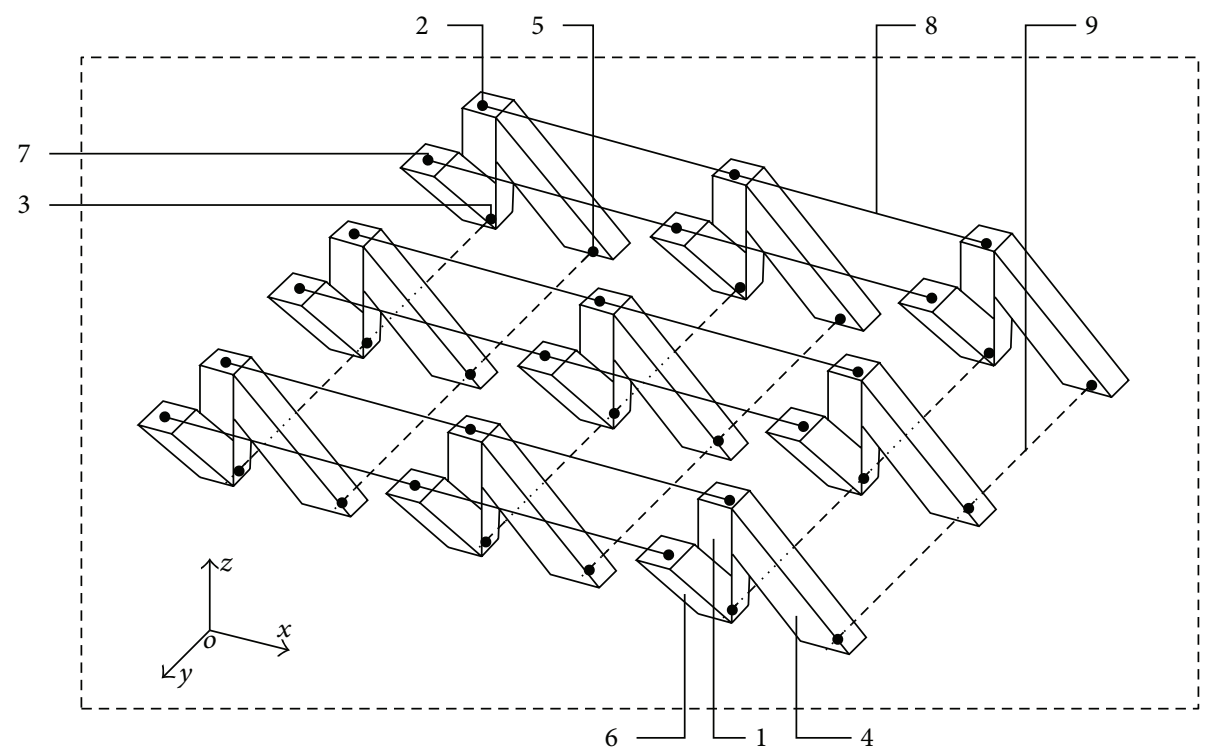

Figure 2: Model of flexible tactile sensor array.

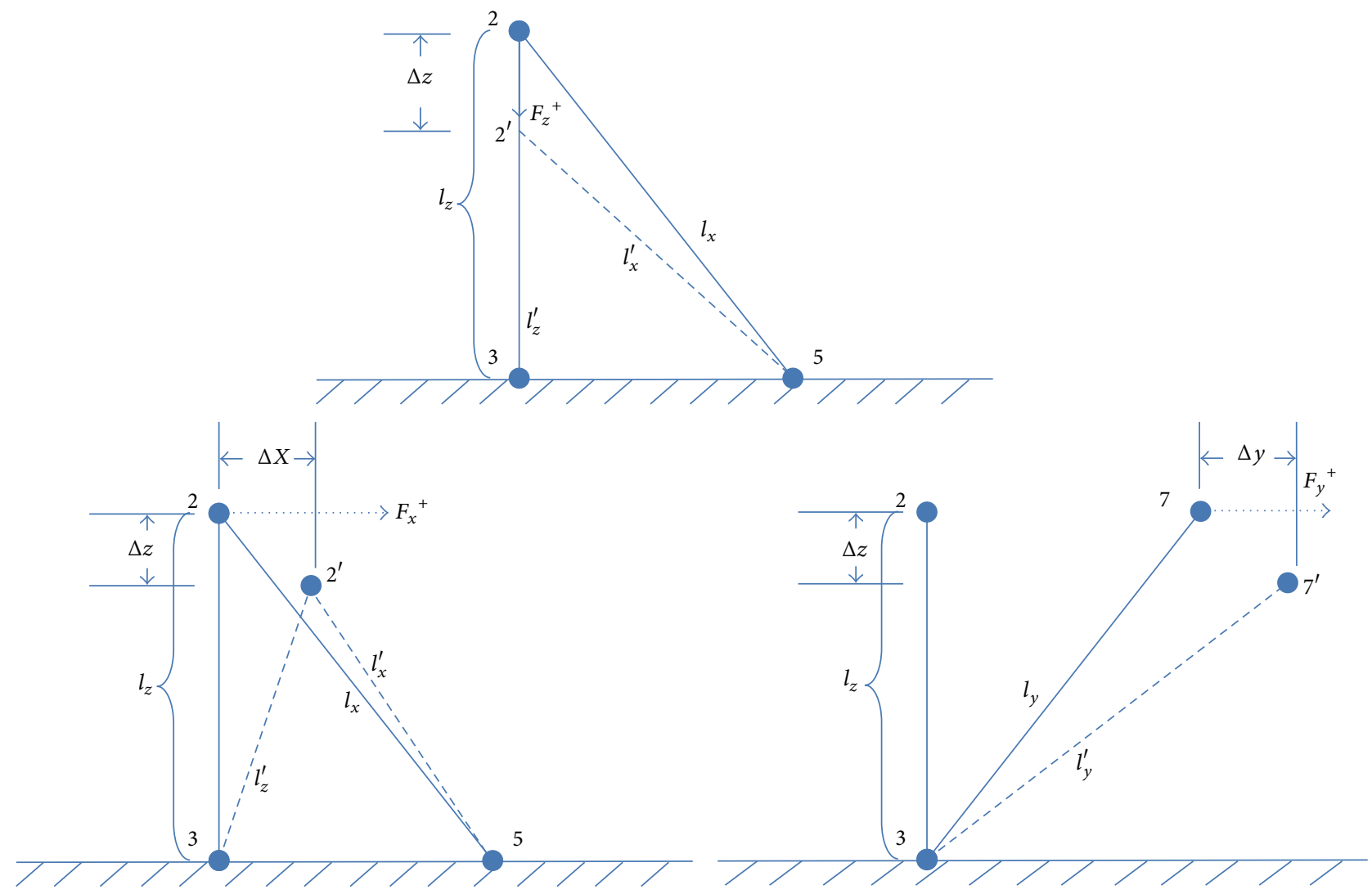

FIGURE 3: Deformation status of the sensitive unit.

which imply that the coordinates of lower sensing electrodes labeled " 3 " and " 5 " are not changed. When external force loaded on the sensor, the upper surface will be deformed. The position of sensing electrodes " 2 " or " 7 " would be changed, and they are moved to $2^{\prime}$ and $7^{\prime}$. The deformation status of the micro structure sensitive unit is shown in Figure 3. We can deduce and solve the length variation of each pillar
$\left(\Delta l_{i}, i=x, y, z\right)$ through the changed resistance by scanning the external circuit firstly and then simulate the deformation (displacement) of the upper electrodes and at last decouple the force loaded on the novel tactile sensor.

It is assumed that when force loaded on the sensor, the volume of each conductive pillar consisting of force-sensitive conductive rubber remains the same. Accordingly, resistances 
of the three conductive pillars of each sensitive unit can be computed by the following formula:

$$
R_{i}=\rho \frac{l_{i}}{S}=\rho \frac{l_{i}^{2}}{V_{i}}, \quad i=x, y, z,
$$

where $V_{x}, V_{y}, V_{z}$ indicate the volumes of conductive pillars labeled "1," " 4 ," and "6," respectively. $l_{x}, l_{y}, l_{z}$ indicate the original length of those conductive pillars. $l_{i}$ and $V_{i}(i=$ $x, y, z)$ are constants; it means that the initial resistances of all the three pillars are already known.

When external force loads on the sensor, the length $\left(l_{i}^{\prime}\right)$ of the conductive pillars can be computed by detecting the corresponding resistance $\left(R_{i}^{\prime}\right)$ between the upper row-wire and the lower column-wire:

$$
l_{i}^{\prime}=\sqrt{\frac{R_{i}^{\prime}}{R_{i}}} \cdot l_{i}, \quad i=x, y, z .
$$

Based on detailed analysis, deformation of the sensitive unit of the sensor could be gained. For each micro structure sensitive unit, there are three output components that are $R_{x}^{\prime}$, $R_{y}^{\prime}$, and $R_{z}^{\prime}$, and the input of the sensitive unit is the force which can be decomposed into three components that are $F_{x}$, $F_{y}$, and $F_{z}$.

It is assumed that the conductive pillar satisfies the Generalized Hooke's law. The relationship between stress and strain is linear, which can be described as

$$
\begin{aligned}
& \sigma=E \cdot \varepsilon, \\
& \sigma=E \cdot \frac{\Delta l}{l},
\end{aligned}
$$

where $\sigma$ denotes stress, $E$ is Young's modulus, and $\varepsilon=\Delta l / l$ denotes strain. Additionally,

$$
E \cdot \frac{\Delta l}{l}=\frac{F}{S}
$$

where $S$ denotes the cross-sectional area of the conductive pillar and $F$ denotes the external force. According to (3) and (4), it is gained that

$$
\Delta l=\frac{F \cdot l}{E \cdot S}
$$

where $E \cdot S$ represents the compressive (tensile) stiffness, $\Delta l=$ $l-l^{\prime}$ or $\Delta l=l^{\prime}-l$, and $l^{\prime}$ denotes the pillar length when it is suffered by stress.

From (5), it is known that

$$
F=\frac{E \cdot S}{l} \Delta l
$$

From (1), it is known that

$$
R_{i}=\rho \frac{l_{i}}{S}=\rho \frac{l_{i}^{2}}{V_{i}}, \quad i=x, y, z .
$$

And then, it is also known that when the conductive pillar suffered by external force, its length would be changed.
The deformation (variation) of conductive pillars can be computed as follows:

$$
\Delta l_{i}=l_{i}-l_{i}^{\prime}=\sqrt{\frac{V_{i}}{\rho}}\left(\sqrt{R_{i}}-\sqrt{R_{i}^{\prime}}\right), \quad i=x, y, z,
$$

where $\Delta l_{x}, \Delta l_{y}, \Delta l_{z}$ denotes the deformation of conductive pillar labeled " 4 ," "6," and " 1 ," respectively. $R_{x}^{\prime}, R_{y}^{\prime}, R_{z}^{\prime}$ denotes the resistance of conductive pillar labeled " 4 ," " 6 ," and " 1 " when it suffered by the external force, respectively.

Then, put (8) into (6), it is gained that

$$
F_{i}=\frac{E \cdot S}{l_{i}} \sqrt{\frac{V_{i}}{\rho}}\left(\sqrt{R_{i}}-\sqrt{R_{i}^{\prime}}\right), \quad i=x, y, z .
$$

So, the relationship between three-dimensional force and resistance of conductive pillar can be computed by (9). Further induce

$$
F_{i}=g_{n}\left(R_{i}^{\prime}\right)=f_{n}\left(\Delta l_{i}\right), \quad i=x, y, z ; n=1,2,3 .
$$

By now, the flexible tactile sensor with its novel design strategy achieves the decomposition of three-dimensional information from its structure. It avoids the direct interference between the electrodes and reduces the complexity and nonlinear degree of the tactile sensor, so that the high dimension information of the sensor could be decoupled quickly and accurately. As the length of conductive pillar of each micro structure is very short and its deformation is very small, which is not more than $20 \%$ of the pillar's length, it can be approximately considered that $\Delta l_{i} \cong \Delta i, i=x, y, z . \Delta i$ is the coordinate variation (deformation) of the upper sensing electrodes along $i$-axis direction.

As we can acquire the resistance of conductive pillars by the external circuit of the sensor, this paper takes full advantage of BPNN with strong function approximation ability to approximate the mapping relationship between resistance and force. Then, directly decouple the force loaded on the sensor by the resistance.

Based on the above analysis, this paper concentrates on the study of the multi-dimensional information decoupling and tries to approximate the high dimensional nonlinear mapping relationship of the tactile sensor array using BPNN, so as to improve the decoupling accuracy and real-time performance.

\section{Decoupling Process}

In this paper, the conductive rubber is regarded as an elastomer with continuous, isotropic, repetitive, and no hysteresis properties. According to superposition principle [20], the mathematical relationship between the three-dimensional force and deformation of elastomer can be linear superimposed. If deformation $D_{1}$ is generated by force $F_{1}$ and deformation $D_{2}$ is generated by force $F_{2}$, accordingly, the deformation $D_{1}+D_{2}$ is generated by force $F_{1}+F_{2}$. In view of this, the situation that only vertical force along $Z$-axis is applied on the novel flexible tactile sensor model is studied in the following simulation. 


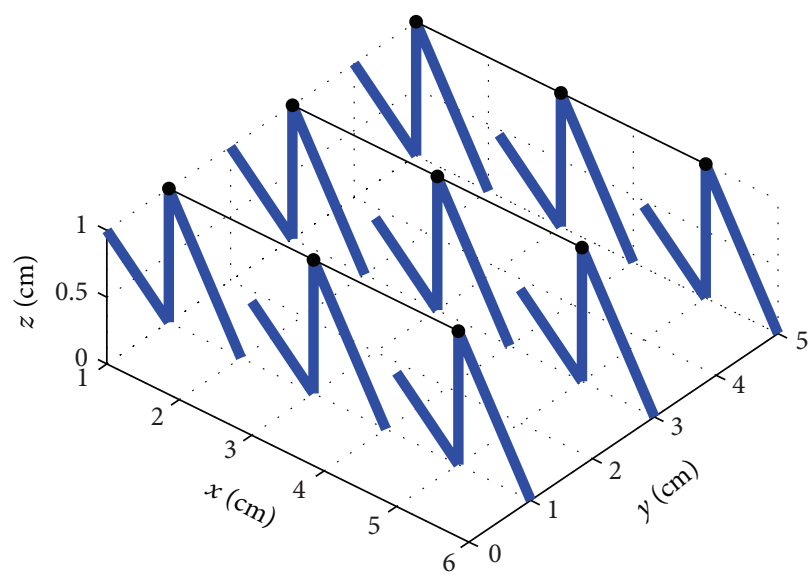

Figure 4: The initial status of the sensor array.

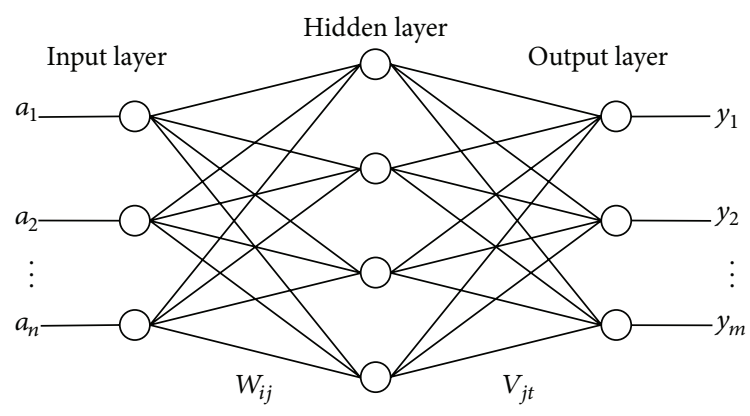

FIgURE 5: The model of BPNN.

In the simulation, the scale of the flexible tactile sensor array based on conductive rubber is $3 \times 3$, which is shown in Figure 4. It is the schematic diagram of the initial status of the sensor array. It depicts the status of the sensor array with no force loaded on. In Figure 4, the black solid circles denote the initial status of the upper sensing electrodes labeled "2." When external force is loaded on the sensor, resistances of conductive pillars can be obtained by scanning the circuit.

In the numerical simulation, the above model is used to generate a set of coordinates (deformation) of the upper electrodes, and then corresponding resistance values of pillars can be detected. We apply COMOL finite element analysis to produce training and testing samples through intelligent analysis and to analyze the feasibility of the numerical model. The resistances are used to get the deformation and force of the sensor via decoupling algorithms. In order to simulate the real deformation of the tactile sensor and improve the decoupling accuracy, we utilize the optimized BPNN algorithm with strong nonlinear approximation ability to decouple the relationship between the resistance values and the force component along $Z$-axis.

3.1. Principle of Back-Propagation Neural Network (BPNN). The BPNN is a widely used multi-layer feed-forward artificial neural network, and it has strong nonlinear mapping capability and good fault tolerance. Normally, the BPNN has three or more layers, which includes an input layer, one or more hidden layers, and an output layer. A three-layer BP network is shown in Figure 5. Each neural node in one layer is connected to all neural nodes in the next layer linking with adjustable weights, and the nodes in the same layer are not connected to each other [21]. When learning samples are put into the network, activation values of each neuron are transmitted from the input layer to the hidden layer and then to the output layer. After that, response of the input signal is gained at the output layer. Next, in accordance with the direction of reducing the error between target output and actual output, the network modifies each connecting weight from the output layer to the hidden layer and then to the input layer. With the modification of counterpropagation error, the accuracy of the BP network is rising.

3.1.1. Learning Process of Standard BP Algorithm. The learning process of standard BP algorithm consists of forwardpropagation of input signal and back-propagation of output error signal [22]. In our simulation, the input signal is the resistance values, the output signal is the force loaded on the sensor.

Forward-Propagation. Input signal is propagated from the input layer to the hidden layer and then to the output layer based on the following formula:

$$
\begin{aligned}
& h_{j}=\varphi_{1}\left(\sum_{i=1}^{n} w_{i j} a_{i}-\theta_{j}\right) \\
& y_{t}=\varphi_{2}\left(\sum_{j=1}^{p} v_{j t} h_{j}-\gamma_{t}\right),
\end{aligned}
$$

where $a_{i}$ is the input signal, $w_{i j}$ is the connecting weight from the input layer to the hidden layer, $v_{j t}$ is the connecting weight from hidden layer to output layer, $\varphi_{1}$ and $\varphi_{2}$ are the activation function of the hidden layer and the output layer, respectively, $h_{j}$ and $y_{t}$ are the output of the hidden layer and the output layer, respectively, and $\theta_{j}$ and $\gamma_{t}$ are the biases of the hidden layer and the output layer, respectively.

Back-Propagation. Output errors are propagated from the output layer to the hidden layer and then to the input layer. During this process, all of the connecting weights $v_{j t}$ and $w_{i j}$ are modified, respectively. If the output error doesn't reach the target value, this process continues to be iterated. In that case, the weights and biases of the output layer are modified as follows:

$$
\begin{gathered}
v_{j t}(N+1)=v_{j t}(N)+\eta \delta_{t}^{y} h_{j}, \\
\gamma_{t}(N+1)=\gamma_{t}(N)+\alpha \delta_{t}^{y},
\end{gathered}
$$

where $v_{j t}(N)$ and $v_{j t}(N+1)$ are the weights from the hidden layer to the output layer of time $N$ and $N+1 ; \gamma_{t}(N)$ and $\gamma_{t}(N+$ 1) are the biases of the output layer in time $N$ and $N+1 ; \alpha$ is 
the learning coefficient and $0<\alpha<1 ; \delta_{t}^{y}$ is the local gradient of the output layer, which is as follows:

$$
\begin{aligned}
\delta_{t}^{y} & =\left(o_{t}-y_{t}\right) \varphi_{2}^{\prime}\left(\sum_{j=0}^{n} h_{j} v_{j t}(N)\right) \\
& =\left(o_{t}-y_{t}\right) y_{t}\left(1-y_{t}\right),
\end{aligned}
$$

where $o_{t}$ is the target output and $y_{t}$ is actual output of the network.

Accordingly, the weights and biases of the hidden layer are modified as follows:

$$
\begin{gathered}
w_{i j}(N+1)=w_{i j}(N)+\beta \delta_{j}^{h} a_{i} \\
\theta_{j}(N+1)=\theta_{j}(N)+\beta \delta_{j}^{h},
\end{gathered}
$$

where $w_{j t}(N)$ and $w_{j t}(N+1)$ are the weights from the input layer to the hidden layer of time $N$ and $N+1 ; \theta_{j}(N)$ and $\theta_{j}(N+1)$ are the biases of the hidden layer in time $N$ and $N+1 ; \beta$ is the learning coefficient, $0<\beta<1$; and $\delta_{j}^{h}$ is local gradient of the hidden layer, which is as follows:

$$
\begin{aligned}
\delta_{j}^{h} & =\left[\sum_{t=1}^{q} \delta_{t}^{y} v_{j t}(N)\right] \varphi_{1}^{\prime}\left(\sum_{i=0}^{m} a_{i} w_{i j}(N)\right) \\
& =\left[\sum_{t=1}^{q} \delta_{t}^{y} v_{j t}(N)\right] h_{j}\left(1-h_{j}\right) .
\end{aligned}
$$

The essence of the BP algorithm is to propagate the error inversely and adjust the weights until the output error achieves a threshold value. That process is based on gradient descent method which minimizes the sum of the squared errors between the actual and the desired output values. The BPNN with a single hidden layer could approximate any continuous function in the closed area with arbitrary accuracy [18]. A three-layer BP network can complete any nonlinear mapping between $n$-dimensional input space and $m$-dimensional output space.

3.1.2. Improved BP Algorithm. There are some shortcomings in the standard BP algorithm described above, such as slow convergence rate and easy convergence to local minimum. In order to improve the BP algorithm, the Levenberg-Marquardt (LM) algorithm with fast convergence rate and strong robusticity on setting the initial value is used to optimize the BP method; it is called LMBP algorithm. The LM algorithm is a numerical optimization algorithm; it originates from the Newton method. It is also the combination of gradient descent method and Newton method. The LMBP algorithm could get fast decline at the beginning of the search and find an ideal search direction at the vicinity of the optimal value. The precision of LMBP is higher than that of Newton method.
The adjustable weight vector and bias of LMBP algorithm are given as follows:

$$
\begin{aligned}
W(N+1) & \\
= & W(N) \\
& -\left[J^{T}(W(N))+\mu(N)\right]^{-1} J^{T}(W(N)) e(W(N)) \\
b(N+1) & \\
= & b(N) \\
& -\left[J^{T}(b(N))+\mu(N)\right]^{-1} J^{T}(b(N)) e(b(N)),
\end{aligned}
$$

where

$$
J(W)=\left[\begin{array}{cccc}
\frac{\partial e_{1}(W)}{\partial w_{1}} & \frac{\partial e_{1}(W)}{\partial w_{2}} & \cdots & \frac{\partial e_{1}(W)}{\partial w_{n}} \\
\frac{\partial e_{2}(W)}{\partial w_{1}} & \frac{\partial e_{2}(W)}{\partial w_{2}} & \cdots & \frac{\partial e_{2}(W)}{\partial w_{n}} \\
\vdots & \vdots & \vdots & \vdots \\
\frac{\partial e_{N}(W)}{\partial w_{1}} & \frac{\partial e_{N}(W)}{\partial w_{2}} & \cdots & \frac{\partial e_{N}(W)}{\partial w_{n}}
\end{array}\right]
$$

is Jacobin matrix and $e(W)$ is error function. $\mu$ is compensation factor. Consider

$$
F(W)=\sum_{i=1}^{N} e_{i}^{2}(W)=e^{T}(W) e(W) .
$$

If a step does not reduce the value of $F(W)$, then $\mu$ will be multiplied by a factor $\eta(\eta>1)$; otherwise, in the next step, the compensation factor $\mu$ will be divided by $\eta$. This process will be repeated. Factor $\mu$ will be modified in each iteration computation which ensure that the LM algorithm has a good stability and fast convergence rate.

In the experiment, LM method is used to improve the standard BPNN. The improved BPNN is used as an approximate machine to approach the high dimensional nonlinear mapping relation from resistance value to force along $Z$-axis. During the decoupling process, the sensor model is viewed as a black box, which generates a large number of training data. The samples are trained by the LMBP neural network as far as possible to approximate the original model equation.

3.2. Construction of the BPNN for the Tactile Sensor Model. Our BPNN contains three layers. The first is the input layer, which is a vector of resistance values corresponding to the force along $Z$-axis. The second is the hidden layer. The number of hidden nodes is variable. In the experiments, the number of the hidden nodes is from 5 to 14 . The last is the output layer, which is a vector of force along $Z$-axis loaded on the tactile sensor. In the numerical simulation, we use MATLAB neural network toolbox to construct BPNNs. tan-sigmoid function and linear function are used as the activation function of the hidden layer nodes and the output layer nodes, respectively. The structure of BP network for the flexible tactile sensor is shown in Figure 6. 


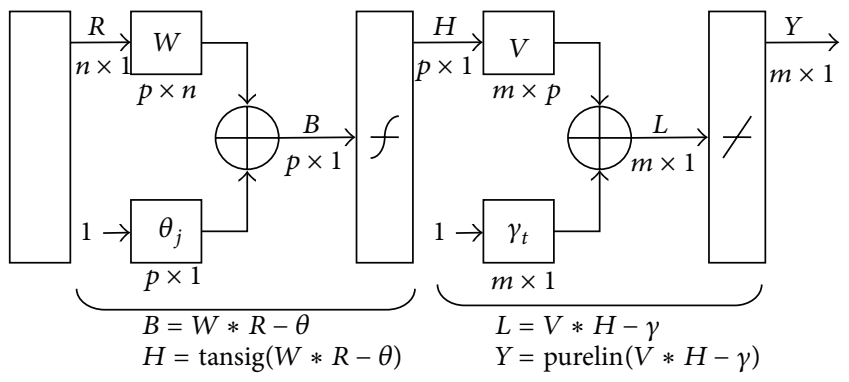

FIGURE 6: BP network structure of the novel tactile sensor.

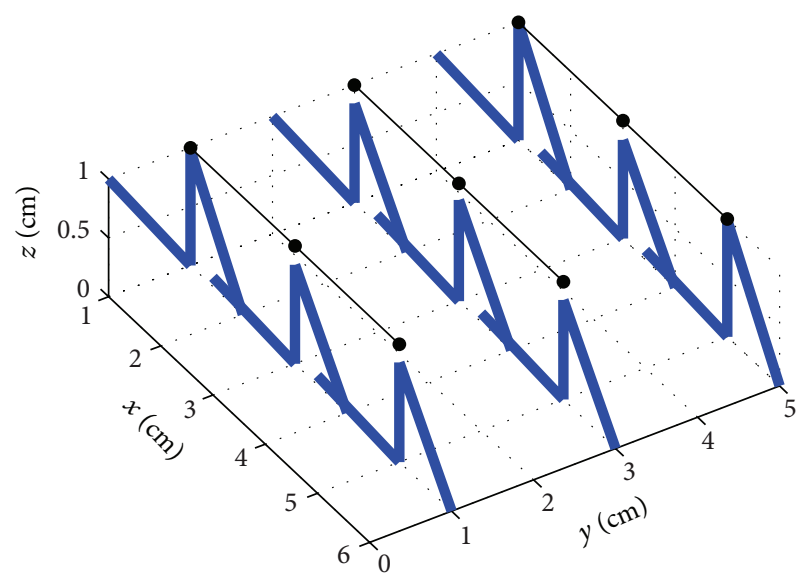

Figure 7: Deformation status of the sensor array.

In Figure 6, $R$ is the input signal of the BPNN, which is an $n$-dimensional vector of the resistance; $B$ is the input of the hidden layer; $H$ is the output of the hidden layer; $L$ is the input of the output layer; $Y$ is the output of the output layer, which is an $m$-dimensional vector of force component; and $\theta_{j}$ and $\gamma_{t}$ are the biases of the hidden layer and output layer. In the simulation, the value of $n$ and $m$ is 9 .

\subsection{Decoupling Process Based on the Improved BP Algorithm.} We decouple the force loaded on the novel flexible tactile sensor using the improved BPNN algorithm through the resistance of conductive pillar. In the experiment, there are 1000 training samples and 100 testing samples. Each sample includes two parts: a 9-dimensional vector of resistance of the pillar labeled "1" and a 9-dimensional vector of force along $Z$-axis. The input signal of each sample for the BPNN is a 9dimensional vector of resistance, which is described as

$$
R=\left[R_{z}^{1}, R_{z}^{2}, \ldots, R_{z}^{9}\right]
$$

where $R_{z}^{k}(k=1,2, \ldots, 9)$ indicates the resistance of pillar " 1 " from the $k$ th micro structure sensitive unit when force is loaded. Namely, vector $R$ includes 9 resistances which come from the 9 sensitive units of the sensor array.

The output signal of each sample for the BPNN is a 9dimensional vector of force along $Z$-axis, which is described as

$$
Y=\left[F_{z}^{1}, F_{z}^{2}, \ldots, F_{z}^{9}\right]
$$

where $F_{z}^{k} \in[0 N, 5 N](k=1,2, \ldots, 9)$ indicates the force applied on the pillar labeled " 1 " of the $k$ th micro structure sensitive unit. Namely, vector $Y$ includes 9 force components along $Z$-axis loaded on the 9 micro structures of the tactile sensor array.

When force along $Z$-axis is loaded on the sensor, the sensing array will be deformed. One of the deformation statuses is shown in Figure 7. In which, the black solid circles denote the initial position of the upper sensing electrodes labeled "2."

When establishing the BPNN, the number of hidden layer nodes is very important for the network's performance, it is generally determined by the designer's experience and repeated tests. More hidden nodes may lead to longer learning time for the BP network, but the error may not be the least. It also may lead to poor fault tolerance, poor generalization performance, and poor robustness. Those mean that the more hidden nodes may not gain the better results. The number of hidden nodes is usually connected with the question itself and the number of input/output nodes directly. Although there is no regular formula to compute the hidden nodes number, the following formula is often used to calculate the optimal number of hidden nodes:

$$
h=\sqrt{i+d}+a
$$

where $i$ is the input nodes number, $d$ is the output nodes number, $a$ is an integer constant at 1 to 10 , and $h$ is the hidden nodes number.

In the experiment, (21) is used to compute the hidden nodes number. After that, we also try to set enough hidden nodes firstly, such as 20 , and then during the iterative training process, the invalid hidden nodes will be deleted. That process is repeated, until the number of hidden nodes no longer changed or the target error is gained.

Under the known premise that $i=9$ and $d=9$, through repeated test of the above methods, we conclude that when $a=6$ and $h=10$ the optimal decoupling results of the sensor array are obtained.

After the BPNN improved by LM algorithm is constructed, it is used to learn and approximate the mapping relationship between the input signal and output signal. 1000 samples are applied to train the improved BP network. It is expected to minimize the errors and obtain the optimal network model. In the training process, the target mean square error is 0.0001 . The 100 testing samples are used to evaluate the performance of the improved BPNN.

The average relative decoupling errors of force along $Z$ axis based on the improved BP algorithm with different hidden nodes number are shown in Table 1. From which, it is known that the more hidden nodes do not always gain better decoupling results. More hidden nodes may lead to poor robustness. Table 1 concludes that the performance with 10 hidden nodes is the best. When the hidden nodes number exceeds 5, the average relative decoupling errors between the actual force and the decoupled force along $Z$-axis decreased quickly. However, when the number exceeds 10, the decoupling errors increased. Those strongly indicate that there exist an optimal number of hidden nodes in the BPNN 
TABLE 1: Average relative decoupling errors.

\begin{tabular}{lc}
\hline Hidden nodes number & AveRelErr $(\%)$ \\
\hline 5 & 3.07 \\
6 & 2.95 \\
7 & 2.63 \\
8 & 2.19 \\
9 & 1.96 \\
10 & 1.72 \\
11 & 1.83 \\
12 & 1.98 \\
13 & 2.17 \\
14 & 2.06 \\
\hline
\end{tabular}

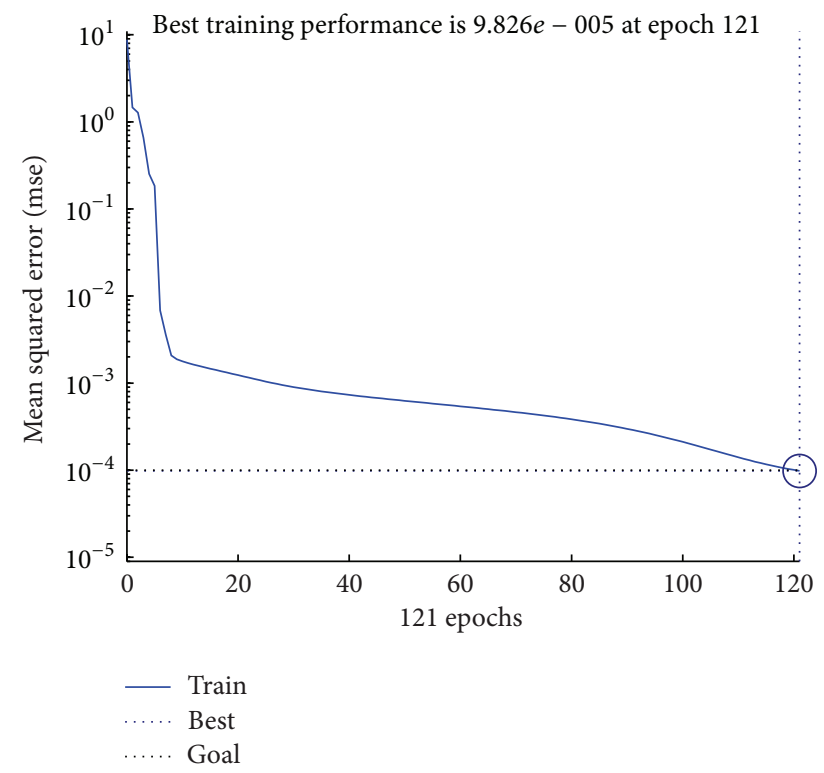

FIGURE 8: The training performance of BPNN.

model. The average relative decoupling errors are computed as follows:

$$
\text { AveRelErr }=\frac{\sum_{t=1}^{9 * \text { Num }}\left|\left(F_{t}^{\prime}-F_{t}\right) / F_{t}\right|}{9 * \text { Num }},
$$

where $F_{t}^{\prime}$ denotes the decoupled result of force component $F_{z}$ for the $t$ th testing sample, $F_{t}$ denotes the actual force component along $Z$-axis applied on the tactile sensor for the $t$ th testing sample, and AveRelErr denotes the average relative decoupling error of the testing samples, Num $=100$ (the number of testing samples). As each sample includes the information of a sensor array consisting of 9 micro structure sensitive units, the 100 testing samples include $9 *$ Num $(900)$ force components.

Figure 8 describes the performance during the training process of the BPNN with 10 hidden nodes. It can be seen that the learning process of the BPNN is finished after 121 times of iteration and reaches the accuracy of 0.0001 . After that, the trained BPNN model is used to decouple the relation between high dimensional input signal and output signal from the 100

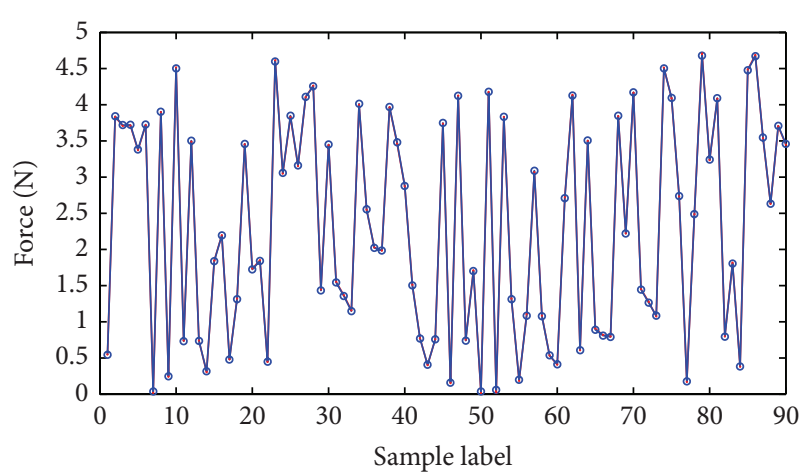

FIGURE 9: Decoupling results.

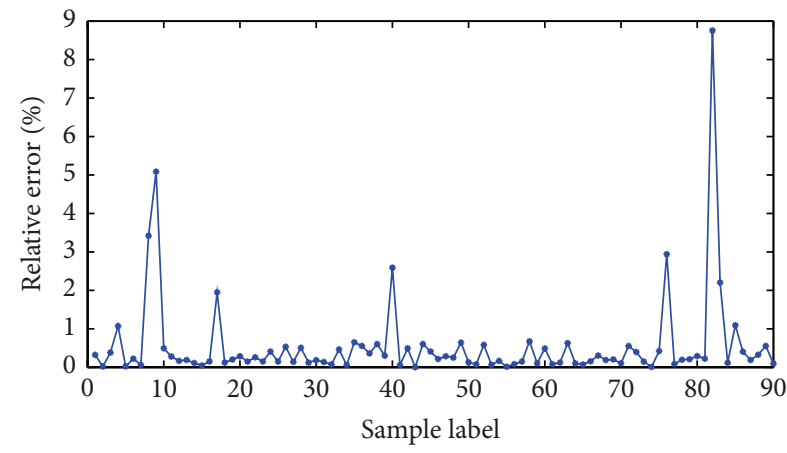

FIGURE 10: Relative decoupling error of the random sample.

testing samples and directly decouple the force exerted on the sensor array. At last, we gain the average relative decoupling error of 900 force components along $Z$-axis.

\section{Analysis and Discussion of Decoupling Results}

10 samples are randomly selected from the 100 testing samples. Figure 9 shows the decoupling results of the 90 force components along $Z$-axis from those random samples. In Figure 9, the red dots represent the actual force component applied on the sensor and the blue circles represent the decoupled force component based on the improved BPNN algorithm. As can be seen from Figure 9, the decoupled result is almost coincident with the actual force, which implies that the decoupling accuracy through the improved BPNN method is very good. The LMBP algorithm we used is very suitable to decouple and approximate the relationship between resistance and force along $Z$-axis for the sensor array.

Figure 10 describes the relative decoupling error of 90 force components from the 10 random testing samples corresponding to those in Figure 9. Each sample includes the information of 9 micro structure sensitive units. Figure 10 shows that the maximum relative error of force along $Z$-axis is not more than $9 \%$, and most of the decoupling errors of pressure are less than $1 \%$. 


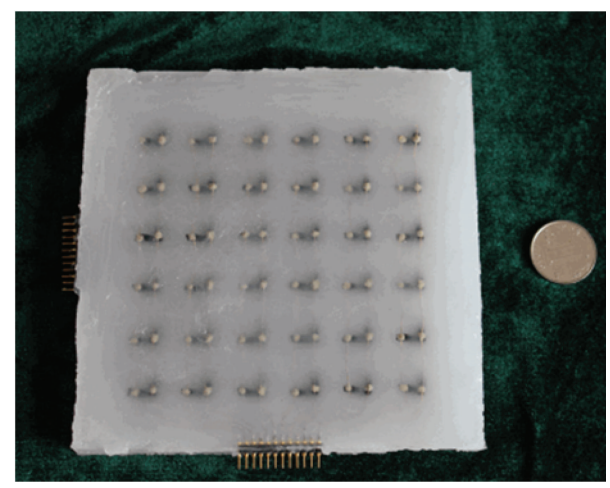

FIgURE 11: A novel flexible tactile sensor prototype.

As can be seen from Figure 10, the relative decoupling errors of several data points at the left and right edge are obviously abnormal, which are much higher than other decoupling errors. There are two main reasons for the abnormal data as follows.

(1) The data points with high relative decoupling error are at the edge of the sensor array, because the effective information the edge point obtained is much less than the other data points, and there usually exists more noise.

(2) The micro structure of the sensor array is composed of conductive rubber which is a kind of nonlinear forcesensitive material with certain field effect in practical application. Therefore, during the signal acquisition process, there exists crosstalk phenomenon. So, in the procedure of acquiring the timely resistance signal when force loaded, there also inevitably exists error.

The above decoupling results are gained based on the improved BPNN with the condition of Goal $=0.0001$ (target mean square error) and $h=10$ (hidden nodes number). All of the decoupling results verify the validity of our method and the high efficient function approximation ability of the improved BPNN algorithm.

After the structure analysis and decoupling research, the tactile sensor based on conductive rubber is produced, whose prototype is shown in Figure 11. Because the research of the tactile sensor is very complex, there is still a lot of work to do. In the future, decoupling research will be done based on the real flexible tactile, and the track of the three-dimensional force is expected to be solved accurately. In view of the strong nonlinear mapping ability of BPPNN, the BP algorithm will play an important role in the next works.

\section{Conclusions}

Theoretical analysis and simulation experiments are performed on the novel flexible tactile sensor, and the relation between resistances of conductive pillars and threedimensional force is deduced. The force component loaded on the tactile sensor is decoupled from the resistance information based on the improved BPNN algorithm through the optimized number of hidden layer nodes.
The novel flexible tactile sensor achieves the decomposition of the three-dimensional information from the structure with its unique design, avoids the direct interference between the electrodes, reduces the complexity and the nonlinearity degree of the sensor model, and improves the decoupling accuracy.

\section{Conflict of Interests}

The authors declare that there is no conflict of interests regarding the publication of this paper.

\section{Acknowledgments}

This work is supported by NSF of Anhui Province (no. 1408085QF123), the NSFC (nos. 61372094, 11471304, and 61201076), the Nature Science Research Project of Universities of Anhui Province (KJ2015JD10), and the Doctor Foundation of Anhui Jianzhu University (2014).

\section{References}

[1] A. Wisitsoraat, V. Patanasetagul, A. Tuantranont, and N. Poonnikorn, "Design and fabrication of non-silicon-based piezoresistive MEMS tactile sensor," in Proceedings of the 5th IEEE Conference on Sensors, pp. 1317-1320, Daegu, Republic of Korea, October 2006.

[2] B. Sümer, B. Aksak, K. Şahin, K. Chuengsatiansup, and M. Sitti, "Piezoelectric polymer fiber arrays for tactile sensing applications," Sensor Letters, vol. 9, no. 2, pp. 457-463, 2011.

[3] H. B. Muhammad, C. Recchiuto, C. M. Oddo et al., "A capacitive tactile sensor array for surface texture discrimination," Microelectronic Engineering, vol. 88, no. 8, pp. 1811-1813, 2011.

[4] M. A. Qasaimeh, S. Sokhanvar, J. Dargahi, and M. Kahrizi, "PVDF-based microfabricated tactile sensor for minimally invasive surgery," Journal of Microelectromechanical Systems, vol. 18, no. 1, pp. 195-207, 2009.

[5] H.-K. Lee, S.-I. Chang, and E. Yoon, "A flexible polymer tactile sensor: fabrication and modular expandability for large area deployment," Journal of Microelectromechanical Systems, vol. 15, no. 6, pp. 1681-1686, 2006.

[6] S. Pirozzi, "Multi-point force sensor based on crossed optical fibers," Sensors and Actuators A: Physical, vol. 183, pp. 1-10, 2012.

[7] T. Sekitani, Y. Noguchi, K. Hata, T. Fukushima, T. Aida, and T. Someya, "A rubberlike stretchable active matrix using elastic conductors," Science, vol. 321, no. 5895, pp. 1468-1472, 2008.

[8] M. Shimojo, A. Namiki, M. Ishikawa, R. Makino, and K. Mabuchi, "A tactile sensor sheet using pressure conductive rubber with electrical-wires stitched method," IEEE Sensors Journal, vol. 4, no. 5, pp. 589-596, 2004.

[9] D. J. Lipomi, M. Vosgueritchian, B. C.-K. Tee et al., "Skin-like pressure and strain sensors based on transparent elastic films of carbon nanotubes," Nature Nanotechnology, vol. 6, no. 12, pp. 788-792, 2011.

[10] M. Asadnia, A. G. P. Kottapalli, J. M. Miao, and M. S. Triantafyllou, "Ultra-sensitive and stretchable strain sensor based on piezoelectric polymeric nanofibers," in Proceedings of the 28th IEEE International Conference on Micro Electro Mechanical Systems (MEMS '15), pp. 678-681, IEEE, Estoril, Portugal, January 2015. 
[11] J. Engel, J. Chen, and C. Liu, "Development of polyimide flexible tactile sensor skin," Journal of Micromechanics and Microengineering, vol. 13, no. 3, pp. 359-366, 2003.

[12] S. Harada, K. Kanao, Y. Yamamoto, T. Arie, S. Akita, and K. Takei, "Fully printed flexible fingerprint-like three-axis tactile and slip force and temperature sensors for artificial skin," ACS Nano, vol. 8, no. 12, pp. 12851-12857, 2014.

[13] X. X. Wu, A. G. Song, and Z. Wang, "The study on static decoupling algorithm for six-axis force sensor and static calibration," Chinese Journal of Sensors and Actuators, vol. 26, no. 6, pp. 851856, 2013.

[14] J. I. Lee, M.-G. Kim, M. Shikida, and K. Sato, "A table-shaped tactile sensor for detecting triaxial force on the basis of strain distribution," Sensors, vol. 13, no. 12, pp. 16347-16359, 2013.

[15] I. Petra, D. J. Holding, K. J. Blow, B. Tam, X. Ma, and P. N. Brett, "The design of a flexible digit towards wireless tactile sense feedback," in Proceedings of the 8th International Conference on Control, Automation, Robotics and Vision (ICARCV '04), pp. 468-473, Kunming, China, December 2004.

[16] S. Q. Chen, H. Y. Wang, L. Du, and J. Li, "Research on a new type of overvoltages monitoring sensor and decoupling technology," IEEE Transactions on Applied Superconductivity, vol. 24, no. 5, 2014.

[17] S. Baglio, G. Muscato, and N. Savalli, "Tactile measuring systems for the recognition of unknown surfaces," IEEE Transactions on Instrumentation and Measurement, vol. 51, no. 3, pp. 522-531, 2002.

[18] J. X. Ding, Y. J. Ge, Y. Wang, and Z. Wang, "A decoupling algorithm based on homotopy theory for 3-D tactile sensor arrays," Sensors \& Transducers, vol. 136, no. 1, pp. 72-82, 2012.

[19] F. L. Wang, F. Shuang, X. Sun et al., "Decoupling research of a three-dimensional force tactile sensor based on radical basis function neural network," Sensors \& Transducers, vol. 159, no. 11, pp. 289-298, 2013.

[20] K. L. Johnson, Contact Mechanics, Cambridge University Press, Cambridge, UK, 1985.

[21] G. Y. Li, "Multi-sensor information fusion based on BP network," in Proceedings of the 6th International Conference on Natural Computation (ICNC '10), pp. 1442-1445, August 2010.

[22] X. Y. Jiang and Y. J. Bao, "Nonlinear errors correction of pressure sensor based on BP neural network," in Proceedings of the International Workshop on Intelligent Systems and Applications (ISA '09), pp. 1-4, IEEE, Wuhan, China, May 2009. 

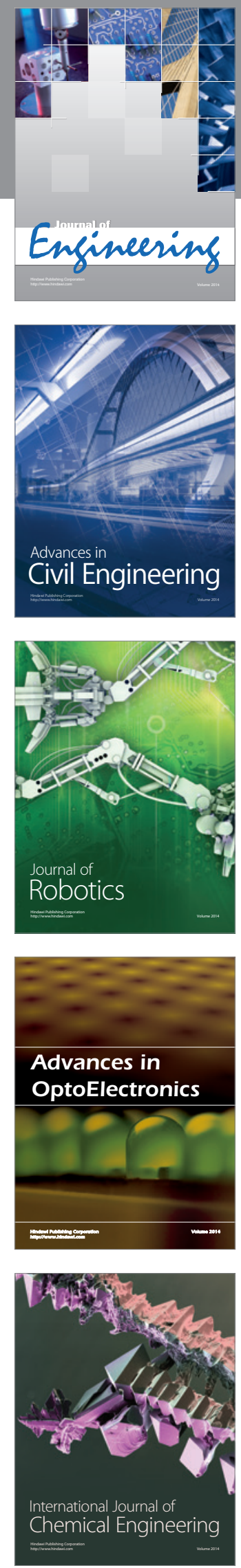

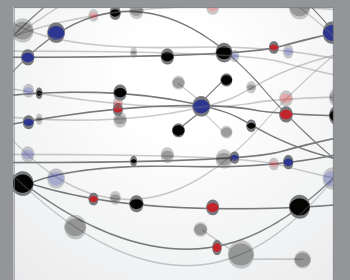

The Scientific World Journal
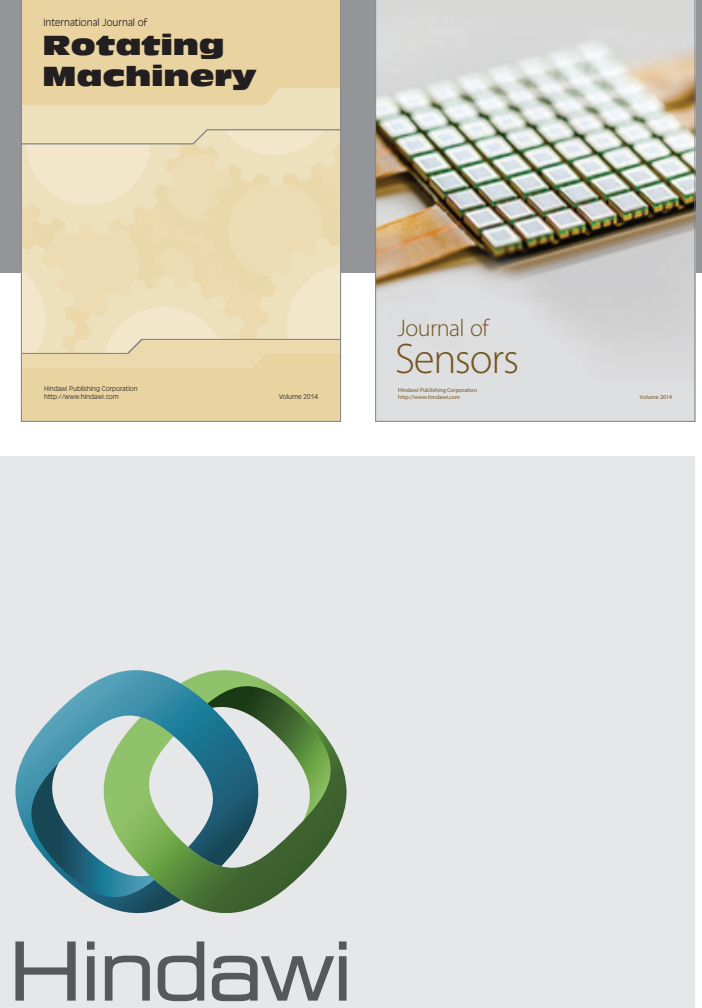

Submit your manuscripts at http://www.hindawi.com
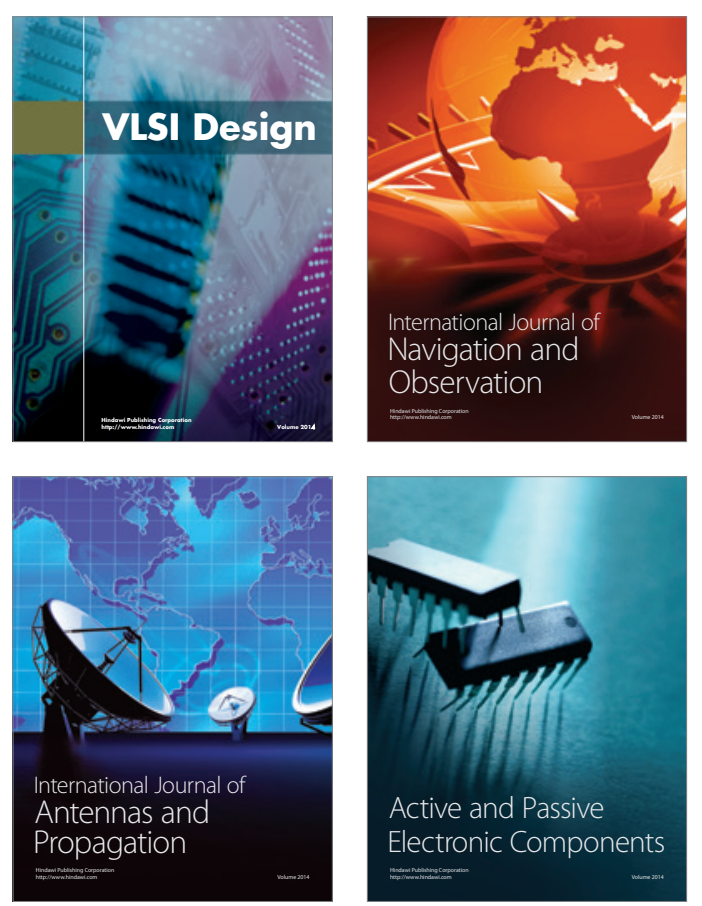
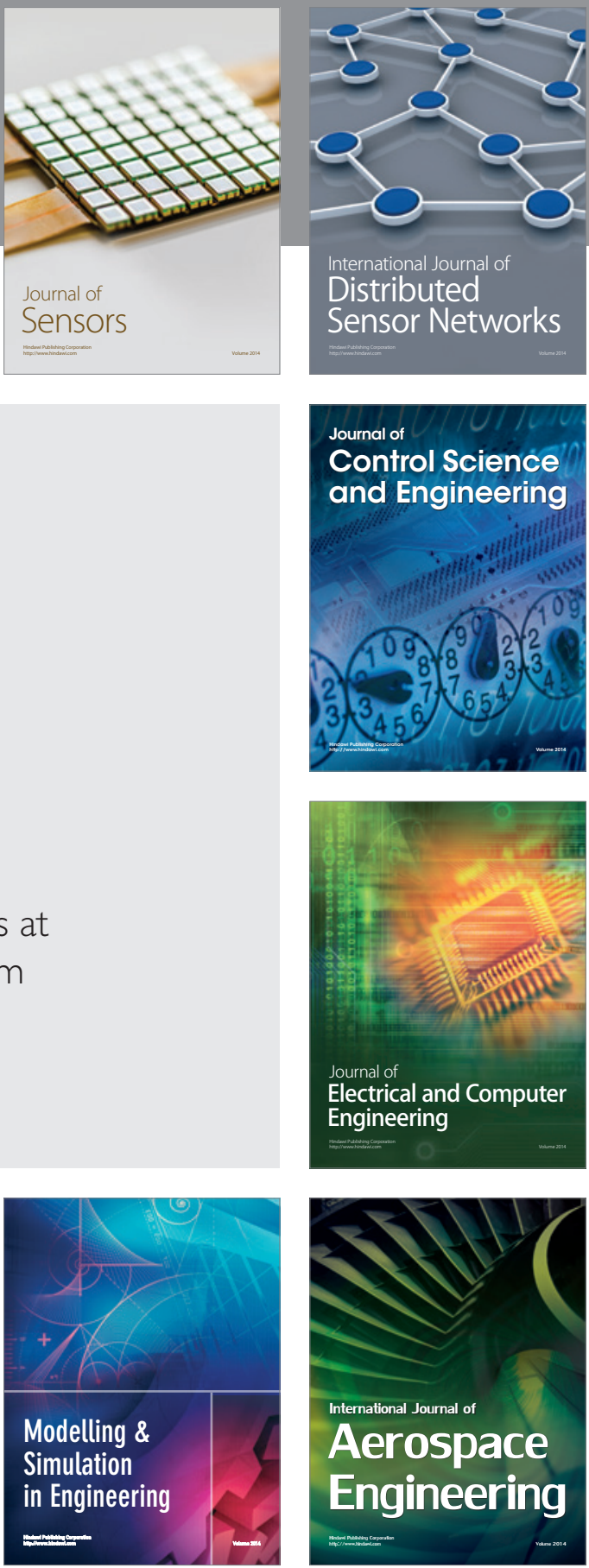

Journal of

Control Science

and Engineering
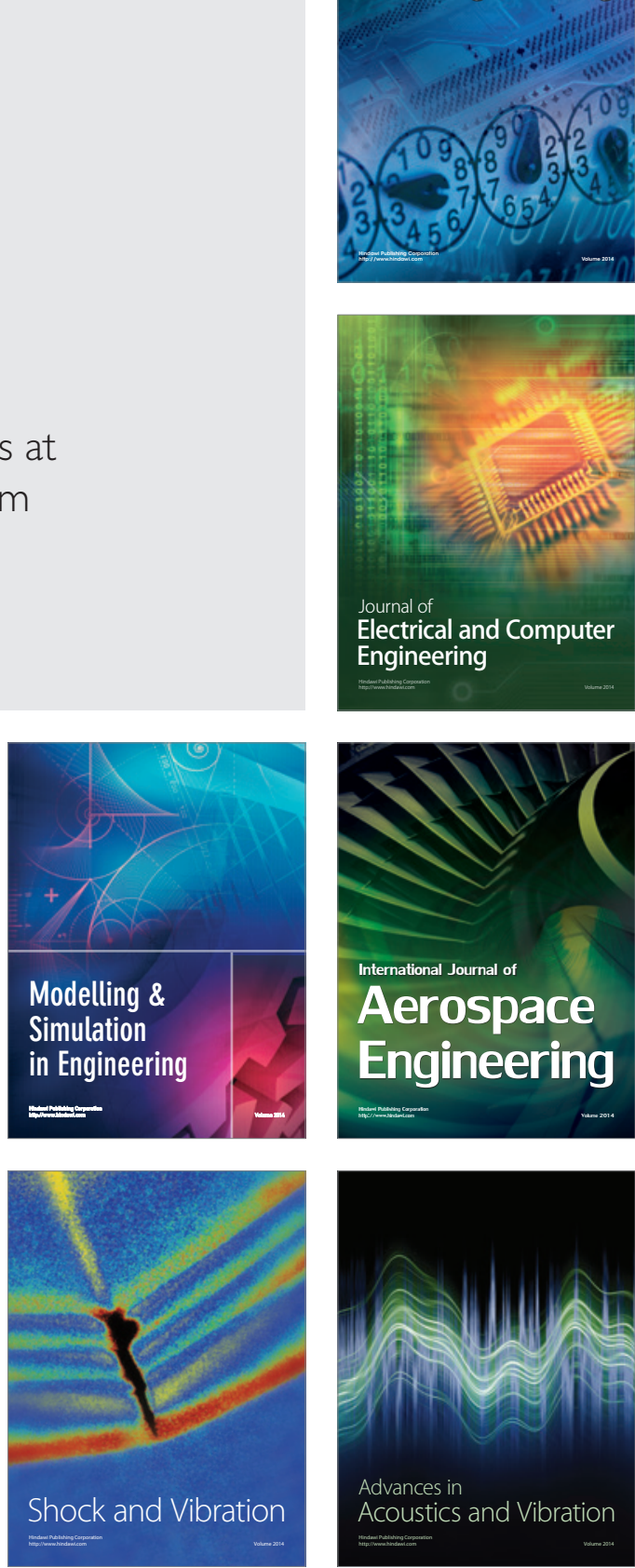